compressive load along its axis is often limited by the risk of buckling; for a given column width and compressive load, higher columns can be built by using materials that have a higher Young's modulus ( $E$, a measure of stiffness). When the height of the column is not limited by an external load, but by just its own weight, then greater heights can be attained using a less dense material: the aim in this context is to maximize the ratio of $E$ to the density $\rho$, rather than just $E$. And when the goal is to build the highest possible column using a fixed mass of material, then it is best to maximize $E / \rho^{2}$. (Maintaining a constant mass is relevant to plants, because synthesizing material is a major cost for them; maximizing $E / \rho^{2}$ corresponds to the most economical way of growing the highest possible column at fixed material costs.)

A consideration of these principles reveals that Song and colleagues' densified wood should perform better than natural, porous wood in the first two scenarios (in which $E$ or $E / \rho$ need to be as large as possible), but only about equally well in the third situation, for which $E / \rho^{2}$ is maximized, on the basis of the changes in stiffness and density reported by the authors. This indicates that trees do not lose much by making wood porous, and that the introduction of pores for water transport comes at no extra material cost. Perhaps because of this, the height of trees is likely to be limited more by hydraulic constraints linked to water transport than by mechanical constraints ${ }^{8}$. Similarly, many advanced-engineering applications require materials that have high stiffness and strength, but in some cases porous materials would increase performance, rather than decrease it.

All biological materials are active, and adapt their internal structure to their function and to environmental needs. Two strategies can be used to repurpose such materials for engineering applications. One is to modify the material to comply with specifications in industrial design, as exemplified by Song et al. with their densification procedure. The other, perhaps more conventional, option is to adapt designs to the properties of natural materials. The latter approach is more sustainable, but would require greater knowledge of how structure relates to function in such materials, and the development of new design approaches ${ }^{9,10}$.

Peter Fratzl is in the Department of Biomaterials, Max Planck Institute of Colloids and Interfaces, Potsdam 14424, Germany. e-mail:fratzl@mpikg.mpg.de

1. Ramage, M. H. et al. Sustain. Energ. Rev. 68, 333-359 (2017)

2. Song, J. et al. Nature 554, 224-228 (2018).

3. Erickson, E. Mechanical Properties of Laminated Modified Wood (US Dept Agriculture, 1965)

4. Ashby, M. F. Metall. Trans. A 14, 1755-1769 (1983).

5. Ashby, M. F., Gibson, L. J.,Wegst, U. \& Olive, R. Proc. R. Soc. Lond. A 450, 123-140 (1995).

6. Gibson, L. J., Ashby, M. F., Karam, G. N., Wegst, U. \& Shercliff, H. R. Proc. R. Soc. Lond. A 450, 141-162 (1995).

7. Fratzl, P. \& Weinkamer, R. Prog. Mater. Sci. 52,
9. Schäffner, W. in +Ultra Knowledge \& Gestaltung (eds Doll, N., Bredekamp, H. \& Schäffner, W.) 23-32

(Seemann, 2017).

10. Fratzl, P. in +Ultra Knowledge \& Gestaltung (eds Doll, N. Bredekamp, H. \& Schäffner, W. 173-178 (Seemann, 2017).

\title{
Many mutations in one clinical-trial basket
}

\section{When abnormality in a gene is linked to cancer and a drug targets the encoded protein, how can the patients who will respond to the drug be identified if the gene is mutated in many different ways in many different cancers? SEE ARTICLE P.189}

\section{ELAINE R. MARDIS}

$\longrightarrow$ ancer usually arises from genomic abnormalities. However, the number and complexity of genetic alterations in tumours can make it difficult to predict whether, and in which tissues, a particular mutation in a specific cancer-linked gene will drive tumour growth. This poses a challenge when trying to identify effective treatments. For example, if a drug that targets a specific protein can treat a person with breast cancer who has a mutation in the gene encoding the protein, could the drug treat another patient who has a different mutation in that gene? And could it treat a person with a mutation in the same gene, but in a tumour that has developed in a different tissue? On page 189, Hyman et al. ${ }^{1}$ report the outcome of a clinical trial testing the ability of the drug neratinib, which inhibits HER2 and HER3 tyrosine kinase enzymes, to reduce or eliminate tumours. The drug was tested on 21 types of cancer in 141 people who had a total of 42 different mutations affecting one of the enzymes.

Studies in the 1970s revealed that certain chromosomal DNA aberrations can be linked to the development of specific cancer types, and that an amplification in the number of copies of particular genes can have a tumourpromoting effect ${ }^{2}$. For example, a highly lethal type of breast cancer is linked ${ }^{3}$ to amplification of the gene ERBB2 and an increase in the level of the HER2 protein that it encodes. HER2 amplification occurs in several other cancers ${ }^{4}$, including colorectal adenocarcinoma and bladder cancer. This understanding led to efforts to develop treatments to stop the action of such overexpressed proteins, resulting in several HER2-targeted therapies that are used in the clinic ${ }^{5}$ to prolong survival in people whose cancers have amplification of ERBB2. Other links between ERBB2 abnormalities and cancer have been identified;

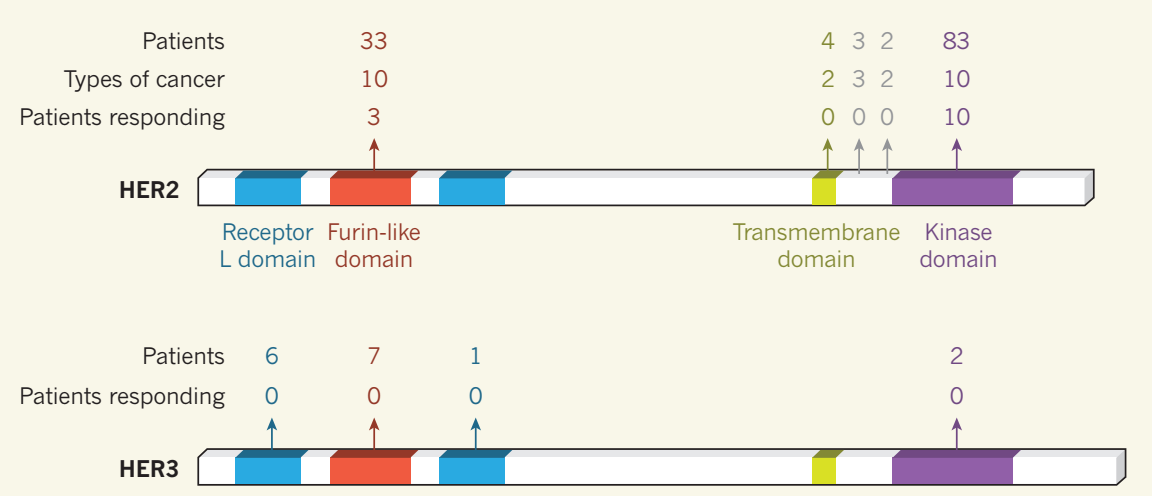

Figure 1 | Results of a cancer clinical trial. Hyman et al. ${ }^{1}$ report the outcome of a study testing how effectively the drug neratinib can treat tumours. The tyrosine kinase enzymes HER2 and HER 3 have been linked to tumour growth and can be inhibited by neratinib. The 141 patients tested had a range of mutations that altered HER2 or HER3, and, between them, had many different tumour types. The protein structures are shown, and arrows indicate the domains or interdomain locations at which protein alterations due to mutations were found. For the HER2 data shown, the cancers were grouped into ten cancer-type categories: biliary, bladder, breast, cervical, colorectal, endometrial, gastro-oesophageal, lung, ovarian or other (for all other cancer types). Responding patient numbers indicate those whose best overall response to the drug was a partial or complete response - a decrease or absence, respectively, of detectable cancer at the end of the trial. 
for example, single-nucleotide mutations in $E R B B 2$ have been found in breast cancers ${ }^{6}$ that do not have amplified $E R B B 2$, and in lung adenocarcinomas ${ }^{7}$.

The rapid development of therapeutics targeting specific cancer-associated proteins has coincided with the rise in DNA sequencing of tumours. In the past decade, the genomic alterations in tens of thousands of cancers have been characterized at single-nucleotide resolution. This has revealed that cancerassociated genes can be altered in myriad ways and that such alterations can be found in primary tumours that arise in many different tissues. However, such variability makes it hard to predict whether a specific drug will have an effect on a patient's cancer; this, in turn, complicates the decision of who to enrol in a clinical trial. One approach to this problem involves introducing the mutated genes in question into preclinical model systems such as genetically engineered mice or cell-line models, but these models are not practical for large-scale investigations of many different gene alterations in different tissue types.

The design of clinical trials testing targeted therapeutics has changed substantially in the era of cancer genomics. Early-phase trials, in particular, now often include people who have an altered target gene, regardless of the tissue in which the tumour is present. These 'basket' trials seek to identify the combination of mutations and tissues that respond to treatment, offering the opportunity, if a trial progresses to a later stage, to focus on tumours in those tissues that are most likely to respond.

The ability of neratinib to target tumours with ERBB2 mutations had been demonstrated $^{6}$ in human-tumour samples transplanted into mice. Hyman et al. used a basket-trial approach to test the effects of the drug on many patients with known tumourdriving ERBB2 mutations; they also examined its effects on a small number of patients who had either rare $E R B B 2$ mutations or mutations in $E R B B 3$, the gene that encodes HER3 and that has also been linked to tumour growth ${ }^{8}$. An interesting feature of the trial design is that it included people with mutations that had not previously been tested for a response to the drug. Some tumour types studied by Hyman and colleagues were not represented in sufficient numbers for the team to assess whether treatment had had a statistically significant effect, and enrolment in the trial is continuing for specific tissues.

The authors found that the effect of neratinib therapy varied in different mutational and tissue contexts. For example, some people who had breast, small-cell lung, cervical, biliary or salivary cancers, and who had certain ERBB2 mutations, responded to the treatment; the greatest effect was observed for breast cancers containing amino-acid alterations in the extracellular or kinase domains of HER2 (Fig. 1). Several patients with previously uncharacterized $E R B B 2$ variants responded to neratinib, supporting the role of these mutations as tumour drivers. Neratinib had no effect on tumours with ERBB3 mutations, nor did it affect colorectal or bladder cancers that had ERBB2 mutations. The bladder-cancer result is consistent with previous studies ${ }^{9,10}$ in which HER2 targeting did not affect this type of cancer. Lack of response to neratinib provides circumstantial evidence that rare alterations in $E R B B 2$ are unlikely to be tumour drivers.

Hyman and colleagues' results indicate that preclinical model studies, such as those suggesting that $E R B B 3$ can drive tumour growth ${ }^{8}$, can sometimes be misleading when trying to infer what happens in a human tumour. This might be because of how the overall genomic context influences the effect of a mutation. A tumour that has an altered target gene could also have alterations in other cancer-promoting genes. Another source of inconsistency between human and mouse studies might be the particular tissue context.

Finally, the genomic heterogeneity of tumour cells (the presence of groups of cells in the tumour that contain different genetic alterations) might be important in determining treatment response. Sequencing analysis conducted by Hyman and colleagues for certain ERBB2 mutations demonstrated that most patients whose $E R B B 2$ mutations were present in all the tumour cells responded to neratinib, whereas those with ERBB2 mutations in only a subset of the tumour cells did not respond.

The authors noted that response to treatment could be affected by the particular genetic mutation, the location of the tumour and the specific pattern of other mutated cancer-associated genes present. This will probably hold true for most, if not all, future basket trials of targeted inhibitor therapies and is quite instructive for such studies. More-complete genomic characterization of tumours, beyond the gene(s) being targeted, will be needed to determine the genomic context linked to response or resistance to treatment. The genomic profiles and therapeutic-response data from basket trials such as this one should be made publicly available as a way of improving the design of clinical trials of other agents. Such data sets might contribute to the development of diagnostics that enable the precise identification of those patients who are most likely to benefit from targeted treatment. The data could also help to streamline the design of clinical trials and thereby hasten cancer therapeutics towards regulatory approval.

Elaine R. Mardis is at the Institute for Genomic Medicine, Nationwide Children's Hospital, Columbus, Ohio 43205, USA. e-mail:elaine.mardis@nationwidechildrens.org

1. Hyman, D. M. et al. Nature 554, 189-194 (2018).

2. Semba, K. et al. Proc. Natl Acad. Sci. USA 82, 6497-6501 (1985)

3. Slamon, D. J. Science 235, 177-182 (1987).

4. Scholl, S., Beuzeboc, P. \& Pouillart P. Ann. Oncol. 12, S81-S87 (2001).

5. Slamon, D. J. et al. N. Engl. J. Med. 344, 783-792 (2001).

6. Bose, R. et al. Cancer Discov. 3, 224-237 (2013).

7. Stephens, P. et al. Nature 431, 525-526 (2004).

8. Jaiswal, B. S. et al. Cancer Cell 23, 603-617 (2013).

9. Oudard, S. et al. Eur J. Cancer 51, 45-54 (2015).

10.Powles, T. et al. J. Clin. Oncol. 35, 48-55 (2017).

This article was published online on 31 January 2018.

\section{CLIMATE SCIENCE}

\section{Fossil-fuel subsidies assessed}

Many governments subsidize the production and consumption of fossil fuels. Contrary to expectation, a study finds that removing these subsidies would only modestly reduce global carbon dioxide emissions. SEE LETTER P.229

\section{IAN PARRY}

1 The 2015 Paris climate agreement was signed by 195 countries, with most pledging to reduce their emissions of carbon dioxide and other planet-warming gases. Many countries have a long history of subsidizing fossil fuels, and it seems logical that removing these subsidies - as the G20 group of nations has agreed to do - would help them to achieve their Paris climate commitments. However, on page 229, Jewell et al. ${ }^{1}$ report a comprehensive and convincing analysis suggesting that reforming these subsidies would cause only a modest reduction in global $\mathrm{CO}_{2}$ emissions. Nevertheless, I think that there is an urgent need for broader reform of fossilfuel prices to fully reflect the costs associated with global warming and other environmental considerations.

Subsidy reform would increase domestic fossil-fuel prices to match the production costs. Its impact on the climate would therefore depend on how energy demand is affected by 